\title{
Comparação do perfil de suscetibilidade entre isolados clínicos de Candida spp. orais e vulvovaginais no Sul do Brasil
}

\author{
Comparison of susceptibility profile among clinical isolates of oral and vulvovaginal Candida spp. \\ in southern Brazil
}

Daniela Dalazen'; Danuza Zanrosso'; Liliane Wanderley'; Nelci Lopes da Silva²; Alexandre Meneghello Fuentefria ${ }^{3}$

unitermos
Cândida
Antifúngicos
Perfil de suscetibilidade

\section{resumo}

Introdução: Nos últimos anos, o oportunismo das candidíases vem se tornando cada vez mais recorrente e de difícil tratamento, principalmente em virtude da emergência de novas espécies, bem como devido à diminuição à suscetibilidade aos antifúngicos. $\mathrm{O}$ sucesso do tratamento das infecções causadas por Candida depende cada vez mais do conhecimento da espécie e do seu perfil de sensibilidade. Objetivos: Esse estudo teve por objetivo realizar uma investigação do perfil de suscetibilidade de amostras de candidíase oral e vulvovaginal, de pacientes residentes no oeste de Santa Catarina, aos antifúngicos anfotericina B, fluconazol e miconazol. Material e métodos: A suscetibilidade de 60 cepas de Candida spp. foi avaliada frente aos antifúngicos. As determinações das concentrações inibitórias mínimas e concentrações fungicidas mínimas foram realizadas com base no documento M27-A2 do Clinical and Laboratory Standards Institute (CLSI). Resultados: No presente estudo foi observado alto índice de resistência para o fluconazol e para a anfotericina $\mathrm{B}$. $\mathrm{O}$ miconazol foi o antifúngico que demonstrou melhor eficácia sobre as cepas testadas. Discussão: As amostras dessa região do estado de Santa Catarina demonstram baixa suscetibilidade aos antifúngicos, o que explica a alta taxa de recidivas nas infecções, mas alerta para a emergência de cepas com resistência adquirida. Conclusão: A prévia avaliação por testes de suscetibilidade in vitro deve nortear a conduta de um tratamento antifúngico efetivo para os casos de candidíase de repetição, principalmente em locais de alta prevalência desta infecção oportunista, como no oeste de Santa Catarina.

\begin{abstract}
Introduction: In recent years, the opportunism of yeast infections is becoming more recurrent and difficult to treat, mainly due to the emergence of new species as well as due to decreased susceptibility to antifungal agents. The successful treatment of infections caused by Candida is increasingly dependent on knowledge of the causative species and its susceptibility profile to antifungals. Objectives: This study aimed to conduct an investigation of the susceptibility profile of samples of oral and vulvovaginal candidiasis in patients of western Santa Catarina to the antifungal amphotericin B, fluconazole and miconazole. Material and methods: The susceptibility of 60 strains of Candida spp. was evaluated for antifungal agents. The determination of minimum inhibitory concentrations and minimal fungicidal concentrations were based on document M27-A2 of the CLSI. Results: In this study, we observed a high rate of resistance to fluconazole and amphotericin B. The miconazole was the antifungal agent significantly more effective on the strains tested. Discussion: The samples in this region of the state of Santa Catarina showed a low susceptibility to antifungal agents, which explains the high rate of relapses in infections, but alert to the emergence of strains with acquired resistance. Conclusion: The preliminary assessment by in vitro susceptibility testing should guide the conduct of effective therapy for cases of recurrent mainly in areas of high prevalence of opportunistic infection, as in western of Santa Catarina.
\end{abstract}

key words

Candida

Antifungal agents

Susceptibility profiles

1. Discente do curso de Farmácia da Universidade Comunitária da Região de Chapecó (UNOCHAPECÓ).

2. Mestra e professora titular do curso de Farmácia da UNOCHAPECÓ.

3. Doutor e professor adjunto da Faculdade de Farmácia da Universidade Federal do Rio Grande do Sul (UFRCS). 


\section{Introdução}

O gênero Candida pertence ao reino Fungi, grupo Eumycota, filo Deuteromycota, classe Blastomycetes e faz parte da família Criptococcacea ${ }^{(5)}$. As principais espécies de interesse clínico neste gênero são Candida albicans, $C$. glabrata, C. krusei, C. parapsilosis e C. tropicalis, entretanto outras espécies emergentes deste gênero têm sido isoladas em diversos casos de candidíase e candidemias ${ }^{(3)}$.

As leveduras do gênero Candida sp. são agentes comensais que colonizam a cavidade bucal de cerca de $30 \%$ a $35 \%$ da população adulta sem evidência de infecção. A candidíase da mucosa oral apresenta formas clínicas variadas, como a candidíase pseudomembranosa, popularmente conhecida como sapinho, além das formas eritematosa, hiperplásica e mucocutânea ${ }^{(7)}$. Os principais fatores relacionados com a infecção oral são a síndrome da imunodeficiência adquirida (SIDA), a má-higiene de prótese dentária, os aparelhos ortodônticos e outras doenças imunossupressoras ${ }^{(15)}$.

A candidíase vulvovaginal ocorre principalmente nas mulheres que estão entre a puberdade e a menopausa. Caracteriza-se por lesões na vulva e na vagina, com presença de prurido, ardência e corrimento vaginal de cor branca ${ }^{(1)}$. É encontrada principalmente em pacientes grávidas, diabéticas, que receberam terapêutica microbiana prolongada ou que fazem uso de contraceptivos orais ${ }^{(9)}$. Estima-se que $75 \%$ das mulheres adultas apresentem pelo menos um episódio de vulvovaginite fúngica em sua vida, sendo que, destas, $40 \%$ a $50 \%$ vivenciam novos surtos e em $5 \%$ a candidíase torna-se recorrente. Por outro lado, 20\% a $25 \%$ das mulheres saudáveis e assintomáticas apresentam culturas positivas para leveduras ${ }^{(8)}$.

As candidíases vulvovaginal e oral são consideradas as duas formas mais comuns de infecção fúngica oportunista, e a transformação da condição assintomática para a sintomática indica uma transição da forma saprófita para a forma patógena ${ }^{(14,15)}$. Numerosos estudos indicavam que $C$. albicans era mais frequente do que as espécies não albicans, respondendo por $80 \%$ a $90 \%$ dos casos. Entretanto, nos últimos anos, tem-se observado um aumento expressivo na frequência das espécies não albicans, principalmente C. glabrata, C. tropicalis e C. guillermondi, indicando uma tendência de mudança na etiologia da candidíase após décadas de predomínio de $C$. albicans ${ }^{(5,6,9)}$.

Atualmente, diversos antifúngicos têm sido indicados no tratamento de candidíases oral e vaginal, no entanto a literatura tem demonstrado a ocorrência de cepas com sensibilidade diminuída e outras resistentes in vivo e in vitro a determinados quimioterápicos ${ }^{(2)}$. A introdução dos antifúngicos azólicos representou grande avanço no tratamento das infecções fúngicas, porém seu amplo emprego como profilaxia para pacientes imunocomprometidos, como no tratamento de leveduroses, tem favorecido a seleção de cepas resistentes aos antifúngicos, principalmente por espécies de C. não albicans ${ }^{(15)}$. O perfil de sensibilidade de Candida spp. aos antifúngicos não é o mesmo nas diferentes populações ou comunidades, o que torna fundamental a identificação do agente etiológico causador da candidíase antes de iniciar a terapêutica empírica, bem como a realização do antifungigrama em alguns indivíduos para conhecer e monitorar a resistência a esses fármacos na população que é atendida ${ }^{(12)}$.

Devido à importância clínica desse tema, e sobretudo à ausência de estudos sobre a avaliação da suscetibilidade aos antifúngicos de cepas oriundas de candidíase oral ou vulvovaginal no estado de Santa Catarina, esse estudo teve por objetivo realizar uma investigação do perfil de resistência aos antifúngicos anfotericina B, fluconazol e miconazol, fármacos usualmente encontrados nas prescrições desta região do Brasil.

\section{Materiais e métodos}

Foram avaliadas 30 amostras isoladas durante quadros de recidiva de candidíase oral e 30 de candidíase vaginal durante o segundo semestre de 2009. As amostras orais foram oriundas de pacientes idosos, com sinais clínicos definidos de candidíase eritematosa, sendo a maioria diabética. As amostras isoladas das secreções vaginais tiveram origem em pacientes imunocompetentes, não portadores do vírus da imunodeficiência humana (HIV).

As determinações da concentração inibitória mínima (CIM) e da concentração fungicida mínima (CFM) foram realizadas baseadas no método de diluição em caldo, de acordo com o protocolo M27-A2 estabelecido pelo Clinical and Laboratory Standards Institute (CLSI). Foram utilizados os antifúngicos anfotericina $B$, fluconazol e miconazol nas concentrações de $100 ; 25 ; 6,25 ; 1,56 ; 0,39$ e 0,097 $\mu \mathrm{g} / \mathrm{ml}$, todos em triplicata. Para os testes de CFM foram utilizadas placas com ágar Sabouraud dextrose.

A interpretação dos resultados das CIMs, efetuada de acordo com o documento do CLSI, considera sensível à anfotericina B CIM $\leq 1 \mu \mathrm{g} / \mathrm{ml}$ e resistente $>1 \mu \mathrm{g} / \mathrm{ml}$. Para o fluconazol, considera-se sensível $\mathrm{CIM} \leq 8 \mu \mathrm{g} / \mathrm{ml}$, sensível dosedependente entre 16 e $32 \mu \mathrm{g} / \mathrm{ml}$ e resistente $\geq 64 \mu \mathrm{g} / \mathrm{m}^{(4)}$. O miconazol não é determinado na tabela do CLSI, por isso foi 
comparado com os valores considerados em outros estudos, como o de Isham e Ghannoum ${ }^{(10)}$, que consideram sensivel $\mathrm{CIM}<8 \mu \mathrm{g} / \mathrm{ml}$, sensível dose-dependente entre 8 e $16 \mu \mathrm{g} / \mathrm{ml}$ e resistente $\geq 16 \mu \mathrm{g} / \mathrm{ml}$.

A escolha dos antifúngicos no estudo foi baseada na prevalência da escolha de terapia para candidíase, pelos profissionais prescritores, na região da amostragem. Foi observado que, esporadicamente, ocorre a prescrição de miconazol de uso tópico, principalmente após recidiva com tratamento ao fluconazol em ambos os tipos de candidíase, o que não corresponde ao fármaco preconizado para tratar a repetição dessa doença no paciente.

\section{Resultados}

Na análise com cepas de Candida spp. oriundas de candidíases orais (Tabela 1), as cepas de C. albicans apresentaram CIMs entre 1,56 e $100 \mu \mathrm{g} / \mathrm{ml}$ para anfotericina $B$, sendo que as CFMs corresponderam à mesma variação. Na suscetibilidade para o fluconazol, as CIMs foram de 25 a $\geq 100 \mu \mathrm{g} / \mathrm{ml}$, tendo também a mesma variação das CFMs. Já as CIMs do miconazol variaram entre 1,56 e $\geq 100 \mu \mathrm{g} / \mathrm{ml}$, e as CFMs variaram entre 6,25 e $\geq 100 \mu \mathrm{g} / \mathrm{ml}$. Da mesma forma, as cepas de C. tropicalis tiveram para anfotericina B CIMs entre 1,56 e $\geq 100 \mu \mathrm{g} / \mathrm{ml}$, sendo que as CFMs tiveram a mesma variação; entretanto, para o fluconazol não houve CIMs e consequente CFMs. O miconazol apresentou CIMs entre 25 e $\geq 100 \mu \mathrm{g} / \mathrm{ml}$, sendo que as CFMs tiveram a mesma variação.

Para as cepas de C. krusei as CIMs de anfotericina B variaram entre $1,56 \mathrm{e} \geq 100 \mu \mathrm{g} / \mathrm{ml}$, correspondendo à mesma faixa para as CFMs. A suscetibilidade frente ao fluconazol teve CIMs de $100 \mathrm{a} \geq 100 \mu \mathrm{g} / \mathrm{ml}$, correspondendo à mesma faixa para as CFMs. Já as CIMs do miconazol variaram entre 25 e $\geq 100 \mu \mathrm{g} / \mathrm{ml}$, também com a mesma suscetibilidade para a CFMs. A única cepa de C. glabrata estudada apresentou CIMs e CFMs de 0,097 $\mu \mathrm{g} / \mathrm{ml}$ para os três antifúngicos.

Segundo a $\mathrm{CLSI}\left({ }^{(4)}\right.$, considera-se sensível à anfotericina $\mathrm{B}$ a cepa de Candida que apresentar CIM $\leq 1 \mu \mathrm{g} / \mathrm{ml}$. Em nosso estudo com isolados de origem oral (Tabela 2), apenas a única cepa testada de $C$. glabrata demonstrou-se sensível a esse antifúngico, pois as demais cepas de outras espécies ( $96,6 \%$ do total) se apresentaram resistentes. Para o fluconazol é considerada sensível a cepa que obtiver $\mathrm{CIM} \leq 8 \mu \mathrm{g} / \mathrm{ml}$, sensível dose-dependente entre 16 e $32 \mu \mathrm{g} / \mathrm{ml}$ e resistente $\geq 64 \mu \mathrm{g} / \mathrm{ml}$. Neste fármaco, para os isolados orais, novamente a única cepa de C. glabrata testada no ensaio foi a sensível; entretanto, cerca de $93 \%$ das outras cepas demonstraram-se resistentes. Para o miconazol, cerca de $27 \%$ das cepas foram sensiveis ao antifúngico, sendo $23,3 \%$ pertencentes à C. albicans e 3,4\% à C. glabrata. No entanto, cerca de $70 \%$ dos isolados restantes foram resistentes.

Já nos isolados de secreções vulvovaginais (Tabela 3), as CIMs de anfotericina B para as cepas de C. albicans variaram de $1,56 \mu \mathrm{g} / \mathrm{ml} \mathrm{a} \geq 100 \mu \mathrm{g} / \mathrm{ml}$, bem como as CFMs na mesma faixa. Entretanto, para o miconazol, as CIMs nesta espécie ficaram entre $25 \mu \mathrm{g} / \mathrm{ml} \mathrm{e} \geq 100 \mu \mathrm{g} / \mathrm{ml}$, acompanhadas das CFMs também. O fluconazol não apresentou CIM para nenhuma cepa das espécies testadas, impossibilitando a determinação das CFMs, pois se verificaram $100 \%$ de resistência. Os isolados de $C$. tropicalis tiveram CIMs para anfotericina B entre $6,25 \mu \mathrm{g} / \mathrm{ml} \mathrm{e} \geq 100 \mu \mathrm{g} / \mathrm{ml}$, com CFMs

Variação das CIMs ( $\mu g / \mathrm{ml})$ e CFMs ( $\mu g / \mathrm{ml})$ de 30 amostras de Candida spp. isoladas de Tabela 1 candidíase oral

\begin{tabular}{|c|c|c|c|c|c|c|}
\hline \multirow[b]{2}{*}{ Leveduras ( $n$ ) } & \multicolumn{2}{|c|}{ Anfotericina B } & \multicolumn{2}{|c|}{ Fluconazol } & \multicolumn{2}{|c|}{ Miconazol } \\
\hline & CIM & CFM & CIM & CFM & CIM & CFM \\
\hline C. albicans (14) & $\begin{array}{c}1,56- \\
100\end{array}$ & $\begin{array}{c}1,56- \\
100\end{array}$ & $\begin{array}{c}25- \\
\geq 100\end{array}$ & $\begin{array}{c}25- \\
\geq 100\end{array}$ & $\begin{array}{l}1,56- \\
\geq 100\end{array}$ & $\begin{array}{l}6,25- \\
\geq 100\end{array}$ \\
\hline C. tropicalis (9) & $\begin{array}{l}1,56- \\
\geq 100\end{array}$ & $\begin{array}{l}1,56- \\
\geq 100\end{array}$ & Não & CIM & $\begin{array}{c}25- \\
\geq 100\end{array}$ & $\begin{array}{r}25- \\
\geq 100\end{array}$ \\
\hline C. krusei (6) & $\begin{array}{l}1,56- \\
\geq 100\end{array}$ & $\begin{array}{l}1,56- \\
\geq 100\end{array}$ & $\begin{array}{l}100- \\
\geq 100\end{array}$ & $\begin{array}{l}100- \\
\geq 100\end{array}$ & $\begin{array}{c}25- \\
\geq 100\end{array}$ & $\begin{array}{c}25- \\
\geq 100\end{array}$ \\
\hline C. glabrata $(1)^{*}$ & 0,097 & 0,097 & 0,097 & 0,097 & 0,097 & 0,097 \\
\hline
\end{tabular}

Os valores correspondem, respectivamente, à menor e à maior CIM de cada droga.

*Não foi possível mostrar a variação da CIM porque havia somente uma amostra dessa espécie.

CIM: concentração inibitória mínima; CFM: concentração fungicida mínima; $\mathrm{n}$ : número de amostras por espécie. 
na mesma faixa. O miconazol, por sua vez, apresentou CIMs entre $25 \mu \mathrm{g} / \mathrm{ml} \mathrm{e} 100 \mu \mathrm{g} / \mathrm{ml}$ e as CFMs foram de $100 \mu \mathrm{g} / \mathrm{ml}$.

Para as cepas de C. krusei, as CIMs de anfotericina B variaram de $6,25 \mu \mathrm{g} / \mathrm{ml} \mathrm{a} \geq 100 \mu \mathrm{g} / \mathrm{ml}$, sendo que as CFMs tiveram a mesma variação. As CIMs do miconazol variaram de $25 \mu \mathrm{g} / \mathrm{ml} \mathrm{a} \geq 100 \mu \mathrm{g} / \mathrm{ml}$, e as CFMs também apresentaram essa variação. As cepas de C. glabrata demonstraram CIMs e CFMs de $1,56 \mu \mathrm{g} / \mathrm{ml}$ a $100 \mu \mathrm{g} / \mathrm{ml}$ para anfotericina B; para o miconazol as CIMs e CFMs variaram de 6,25 a $100 \mu \mathrm{g} / \mathrm{ml}$.

Segundo o $\mathrm{CLSI}$, considera-se sensível à anfotericina $\mathrm{B}$ a cepa de Candida que apresentar CIM $\leq 1 \mu \mathrm{g} / \mathrm{ml}$. Neste sentido, os isolados de origem vaginal em nosso estudo apresentaram $100 \%$ de resistência para a anfotericina B e para o fluconazol (Tabela 4). Para o miconazol apenas cerca de $7 \%$ dos isolados se demonstraram sensíveis, representados por cepas de C. krusei e C. glabrata; entretanto, cerca de $93 \%$ apresentaram resistência ao antifúngico.

A análise estatística dos dados foi baseada no teste não paramétrico de Kruskal-Wallis, indicando que não houve diferença significativa nas medidas de CIMs e CFMs dos antibióticos entre os isolados da mesma espécie.

\section{Tabela 2 candidíase oral}

\begin{tabular}{|c|c|c|c|c|c|c|c|c|}
\hline \multirow[b]{2}{*}{ Leveduras ( $n$ ) } & \multicolumn{2}{|c|}{ Anfotericina B } & \multicolumn{3}{|c|}{ Fluconazol } & \multicolumn{3}{|c|}{ Miconazol } \\
\hline & $\begin{array}{c}S \\
(\%)\end{array}$ & $\begin{array}{c}R \\
(\%)\end{array}$ & $\begin{array}{c}S \\
(\%)\end{array}$ & $\begin{array}{c}\text { S-DD } \\
(\%)\end{array}$ & $\begin{array}{c}R \\
(\%)\end{array}$ & $\begin{array}{c}S \\
(\%)\end{array}$ & $\begin{array}{c}\text { S-DD } \\
(\%)\end{array}$ & $\begin{array}{c}\text { R } \\
(\%)\end{array}$ \\
\hline C. albicans (14) & $0(0)$ & $14(46,6)$ & $0(0)$ & $1(3,4)$ & $13(43,3)$ & $7(23,3)$ & $0(0)$ & $7(23,3)$ \\
\hline C. tropicalis (9) & $0(0)$ & $9(30)$ & $0(0)$ & $0(0)$ & $9(30)$ & $0(0)$ & $0(0)$ & $9(30)$ \\
\hline C. krusei (6) & $0(0)$ & $6(20)$ & $0(0)$ & $0(0)$ & $6(20)$ & $0(0)$ & $0(0)$ & $6(20)$ \\
\hline C. glabrata $(1)^{*}$ & $1(3,4)$ & $0(0)$ & $1(3,4)$ & $0(0)$ & $0(0)$ & $1(3,4)$ & $0(0)$ & $0(0)$ \\
\hline Total (30) & $1(3,4)$ & $29(96,6)$ & $1(3,4)$ & $1(3,4)$ & $28(93,3)$ & $8(26,7)$ & $0(0)$ & $22(73,3)$ \\
\hline
\end{tabular}

S: sensível; $S-D D$ : sensível dose-dependente; $R$ : resistente; $n=$ número de amostras por espécie.

\section{Variação das CIMs $(\mu \mathrm{g} / \mathrm{ml})$ e CFMs $(\mu \mathrm{g} / \mathrm{ml})$ de 30 amostras de Candida spp. isoladas de}

Tabela 3 candidíase vulvovaginal

\begin{tabular}{|c|c|c|c|c|c|c|}
\hline \multirow[b]{2}{*}{ Leveduras (n) } & \multicolumn{2}{|c|}{ Anfotericina B } & \multicolumn{2}{|c|}{ Fluconazol } & \multicolumn{2}{|c|}{ Miconazol } \\
\hline & CIM & CFM & CIM & CFM & CIM & CFM \\
\hline C. albicans (17) & $1,56-\geq 100$ & $1,56-100$ & & & $25-\geq 100$ & $25-\geq 100$ \\
\hline C. tropicalis (4) & $6,25-\geq 100$ & $6,25-\geq 100$ & & & $25-\geq 100$ & 100 \\
\hline C. krusei (6) & $6,25-\geq 100$ & $6,25-\geq 100$ & & & $6,25-\geq 100$ & $100-\geq 100$ \\
\hline C. glabrata (3) & $1,56-100$ & $1,56-100$ & & & $6,25-100$ & $6,25-100$ \\
\hline
\end{tabular}

Os valores correspondem, respectivamente, à menor e à maior CIM de cada droga.

CIM: concentração inibitória mínima; CFM: concentração fungicida mínima; n: número de amostras por espécie.

\section{Distribuição do perfil de suscetibilidade aos diferentes antifúngicos de amostras de}

Tabela 4 candidíase vulvovaginal

\begin{tabular}{|c|c|c|c|c|c|c|c|c|}
\hline \multirow[b]{2}{*}{ Leveduras ( $n$ ) } & \multicolumn{2}{|c|}{ Anfotericina B } & \multicolumn{3}{|c|}{ Fluconazol } & \multicolumn{3}{|c|}{ Miconazol } \\
\hline & $\mathrm{S}(\%)$ & $\mathrm{R}(\%)$ & S (\%) & S-DD (\%) & $\mathrm{R}(\%)$ & $\mathrm{S}(\%)$ & S-DD (\%) & $\mathrm{R}(\%)$ \\
\hline C. albicans (17) & $0(0)$ & $17(56,7)$ & $0(0)$ & $0(0)$ & $17(56,7)$ & $0(0)$ & $0(0)$ & $17(56,7)$ \\
\hline C. tropicalis (4) & $0(0)$ & $4(13,3)$ & $0(0)$ & $0(0)$ & $4(13,3)$ & $0(0)$ & $0(0)$ & $4(13,3)$ \\
\hline C. krusei (6) & $0(0)$ & $6(20)$ & $0(0)$ & $0(0)$ & $6(20)$ & $1(3,4)$ & $0(0)$ & $5(16,6)$ \\
\hline C. glabrata (3) & $0(0)$ & $3(10)$ & $0(0)$ & $0(0)$ & $3(10)$ & $1(3,4)$ & $0(0)$ & $2(6,6)$ \\
\hline Total (30) & $0(0)$ & 30 (100) & $0(0)$ & $0(0)$ & 30 (100) & $2(6,8)$ & $0(0)$ & $28(93,2)$ \\
\hline
\end{tabular}

S: sensível; S-DD: sensível dose-dependente; $R$ : resistente; $n=$ número de amostras por espécie. 


\section{Discussão}

A resistência aos antifúngicos em leveduras, assim como qualquer outro microrganismo, pode ser qualificada como intrínseca, natural ou adquirida. A resistência intrínseca caracteriza-se quando todos os membros da espécie são resistentes a certa droga ou classe de agente antifúngico antes de serem expostos ao fármaco, devido à expressão genética dessa condição. A resistência natural, por sua vez, caracteriza-se quando algumas cepas de determinada espécie apresentam resistência. Já a resistência secundária ou adquirida se desenvolve em resposta à exposição a uma droga ${ }^{(11,17)}$. Nos resultados encontrados em nosso estudo, observa-se um predomínio da resistência adquirida, pois boa parte dos isolados clínicos é oriunda de pacientes que foram tratados, mas recidivaram à candidíase. A esperada resistência intrínseca nas cepas identificadas como $C$. krusei foi confirmada, embora se tenha percebido nítida diferença no grau de resistência entre os isolados.

Estudos de isolados de C. albicans provenientes de pacientes com infecções recorrentes têm mostrado que a resistência ao fluconazol ocorre nas mesmas infecções em que anteriormente as cepas eram suscetíveis. Isso confirma os dados de estudos anteriores de que as linhagens são modificadas ao longo da exposição do paciente à droga, caracterizando a resistência adquirida ${ }^{(16)}$.

No presente estudo, nos isolados de Candida spp. de origem oral, vindos de pacientes idosos com sinais clínicos definidos de candidíase eritematosa, o perfil de resistência obtido foi cerca de $97 \%$ para a anfotericina B, 93\% para o fluconazol e $73 \%$ para o miconazol. Embora estudos com isolados de infecções recidivadas não sejam frequentes, a comparação com a resistência encontrada em outros estudos demonstrou o elevado percentual caracterizado em nosso estudo. Lyon et al. ${ }^{(12)}$, em estudo com indivíduos sadios no estado de Minas Gerais, observaram, num total de 109 isolados de Candida spp., cerca de $10 \%$ de resistência ao fluconazol e inibição completa pela anfotericina B. Embora na amostragem os indivíduos não apresentassem sinais de candidíase e muito menos alguma deficiência imunológica predisponente, observava-se uma nítida diferença nos percentuais de resistência, considerando que as amostras em nosso estudo são também de indivíduos sadios, mas com candidíase oral diagnosticada e isolados após recidiva da doença. De forma semelhante aos resultados observados no perfil de suscetibilidade aos isolados de origem oral, nos testes realizados com cepas de infecções recidivadas de candidíase vaginal, obtidas de pacientes imunocompetentes, a resistência foi de $100 \%$ para anfotericina B e para o fluconazol, e cerca de $93 \%$ para o miconazol. Santos et al. ${ }^{(17)}$, por exemplo, evidenciaram, em seu estudo de isolados de candidúria, um percentual de $92 \%$ de resistência ao fluconazol, corroborando o nosso elevado percentual de resistência encontrado para o fluconazol nas amostras vaginais, embora a amostra utilizada nesse estudo seja urina. Entretanto, Ferrazza et al. ${ }^{(8)}$, também no estado de Santa Catarina, porém na região nordeste, observaram absoluta sensibilidade para anfotericina B e para o fluconazol num estudo com mulheres imunocompetentes, tanto em isolados de C. albicans como de não albicans. Essa diferença de percentual dentro do mesmo estado fortalece o entendimento de que o perfil de suscetibilidade dos isolados, quando comparado entre indivíduos com fatores predisponentes semelhantes, tem influência da região de estudo na população amostrada.

A resistência ao miconazol, embora pouco estudada, bem como ainda não estabelecida pelo protocolo do CLSI, tem sido demonstrada nos últimos anos em diversos isolados clínicos de Candida. Num estudo recente com um total de 150 cepas de C. albicans, C. krusei, C. glabrata, C. tropicalis, C. parapsilosis e C. dubliniensis, que representam as mais comumente encontradas tanto em candidíase oral como vulvovaginal, foi observado um percentual de $16 \%$ de resistência, cinco vezes menor que o de nosso estudo ${ }^{(10)}$.

Em geral, para todas as espécies de Candida avaliadas no nosso estudo, o índice de resistência encontrado foi superior ao encontrado por outros autores, o que explica a alta taxa de recidivas das candidíases na região. Apesar de os testes de suscetibilidade terem demonstrado alto índice de resistência aos derivados azólicos, e sabendo-se que em casos de recidiva a conduta padrão adotada pelos clínicos é a prescrição de itraconazol, pode-se concluir que o tratamento com miconazol aplicado por alguns profissionais apresenta menor resistência do que o tratamento com fluconazol e anfotericina B. Embora a utilização do miconazol seja esporádica pelos profissionais da região em recidivas de candidíase após o uso de fluconazol, os resultados desse estudo possibilitam esclarecer que a escolha por miconazol não é a melhor decisão, podendo ser opção no primeiro tratamento, reservando o itraconazol para situações de pacientes que recidivaram. Segundo Leão Ribeiro et al.(11), nenhum dos antifúngicos disponíveis no mercado representa a droga ideal para o tratamento de candidíases. Assim, conclui-se que a combinação de conhecimentos 
farmacológicos de cada antifúngico, associada à resposta terapêutica em cada paciente, e o prévio conhecimento do perfil de suscetibilidade in vitro na população atendida por cada hospital devem nortear a conduta de um tratamento antifúngico mais eficaz para a candidíase. Essa conduta na terapia torna-se cada dia mais indispensável, principalmente em regiões com alta taxa de relatos de recidiva e ineficácia do tratamento, como no caso deste estudo.

Considerando que os testes de sensibilidade aos antifúngicos não são solicitados rotineiramente e que a presente pesquisa detectou um percentual bastante expressivo de leveduras resistentes, sugere-se a necessidade de um programa de monitoramento de amostras de candidíase de repetição na região oeste de Santa Catarina, com o objetivo de acompanhar e demarcar a prevalência do perfil de suscetibilidade.

\section{Agradecimentos}

Agradecemos ao professor Carlos Maxmiliano Dutra, da Universidade Federal do Pampa, pela avaliação estatística dos resultados deste estudo.

\section{Referências}

1. ANDRIOLI, J. L. et al. Frequência de leveduras em fluido vaginal com e sem suspeita clínica de candidíase vulvovaginal. Rev Bras Ginecol Obstet, v. 31, n. 4, p. 300-4, 2009

2. CANDIDO, R. C.; AZEVEdO, R. V. P.; KOMESU, M. C. Leveduras do gênero Candida na cavidade bucal com e sem lesão: detecção de susceptibilidade a antifúngicos - técnica do Etest. J Bras Patol, v. 35, n. 4, p. 210-6, 1999.

3. CARDOSO, B. C. Efeito de antifúngicos em suspensões de biofilmes de Candida albicans e Candida dubliniensis. 2004. 85f. Dissertação (Mestrado em Biotecnologia Engenharia de Bioprocessos) - Escola de Engenharia e Universidade do Minho, Portugal. 2004.

4. CLINICAL AND LABORATORY STANDARDS INSTITUTE. Método de Referência para Testes de Diluição em Caldo para a Determinação da Sensibilidade a Terapia Antifúngica das Leveduras. Norma aprovada. 2. ed. Norma M27-A2 do NCCLS. Estados Unidos, 2002.

5. COLOMBO, A. L.; GUIMARÃES, T. Epidemiologia das infecções hematogênicas por Candida spp. Rev Soc Bras Med Trop, v. 36. n. 5, p. 599-607, 2003.

6. CROCCO, E. I. et al. Identificação de espécies de Candida e susceptibilidade antifúngica in vitro: estudo de 100 pacientes com candidíases superficiais. An Bras Dermatol, v. 79, n. 6. p. 689-97, 2004.

7. EGUSA, H. et al. Oral candidosis in HIV-infected patients. Curr HIV Res, v. 6, n. 6, p. 485-99, 2008.

8. FERRAZZA, M. H. S. H. et al. Caracterização de leveduras isoladas da vagina e sua associação com candidíase vulvovaginal em duas cidades do sul do Brasil. Rev Bras Ginecol Obstet, v. 27, n. 2, p. 58-63, 2005.

9. HOLANDA, A. A. R. et al. Candidíase vulvovaginal: sintomatologia, fatores de risco e colonização anal concomitante. Rev Bras Ginecol Obstet, v. 29, n. 3, p. 3-9, 2007.

10. ISHAM, N.; GHANNOUM, M. A. Antifungal activity of miconazole against recent Candida strains. Mycoses, 2010, [Epub ahead of print].
11. LEÃO RIBEIRO, E. et al. Aspectos das leveduras de Candida vinculadas às infecções nosocomiais. NewsLab, v. 64, n. 3, p. 106-28, 2004.

12. LYON, J. P. et al. Antifungal suscepitibility profile of Candida spp. oral isolates obtained from denture wearers. Braz J Microbiol, v. 39, n. 4, p. 668-72, 2008.

13. WINGETER, M. A. et al. Identificação microbiológica e sensibilidade in vitro de Candida isolada da cavidade oral de indivíduos HIV positivos. Rev Soc Bras Med Trop, v. 40, n. 3, p. 272-6, 2007.

14. PIROFSKI. L.; CASADEVALL. A. Rethinking T cell immunity in oropharyngeal candidiases. J Exp Med, v. 206, n. 2, p. 269-73, 2009.

15. RODRIGUES, G. M. C. et al. Estudo de colonização por Candida sp. na cavidade oral de indivíduos soropositivos e soronegativos para HIV-1 no noroeste Paulista, Brasil. Rev Panam Infect, v. 9, n. 3, p. 26-31, 2007.

16. RODRIGUEZ-TUDELA, J. L. et al. EUCAST breakpoints for antifungals. Drug News Perspect, v. 23, n. 2, p. 93-7, 2010.

17. SANTOS, L. S. et al. Perfil de sensibilidade de amostras isoladas de casos de candidúrias hospitalares aos antifúngicos convencionais. XIII Encontro LatinoAmericano de Iniciação Científica e IX Encontro Latino Americano de Pós-Graduação (Universidade do Vale do Paraíba) 2009.

18. ZARDO, V.; MEZARRI, A. Os antifúngicos nas infecções por Candida sp. NewsLab, v. 63, p. 136-46, 2004.

Endereço para correspondência

Alexandre Meneghello Fuentefria

Departamento de Análises

Universidade Federal do Rio Crande do Sul

Avenida Ipiranga, 2.752 - Bairro Santana

CEP: 90610-000 - Porto Alegre-RS

e-mail: alexmf77@gmail.com 